\title{
Analysis of the appropriateness of orthopaedic computed tomography scans as exemplified by lower extremity bones and joints
}

\author{
Tomasz Latos ${ }^{1, A, B, C, D, E, F}$, Paweł Sztwiertnia ${ }^{1, B, C, D, F}$, Wojciech Wierzchołowski ${ }^{2, D, F}$, Jerzy Michał Walecki ${ }^{1, A, B, C, D, E, F}$ \\ 'Department of Radiology, Medical Centre for Postgraduate Education, Warsaw, Poland \\ ${ }^{2}$ Department of Radiology, Medical College, University of Zielona Góra, Zielona Góra, Poland
}

\section{Abstract}

Purpose: The objective of this study is to analyse the appropriateness of lower extremity coputed tomography (CT) scans as performed in a large orthopaedic hospital.

Material and methods: A total of 1410 CT scans acquired in the years 2014-2018 were analysed for compliance with the "Guidelines for Physicians Issuing Diagnostic Imaging Referrals" (iRefer). These guidelines were published by the Royal Radiologist Society and recommended for use by the Polish Medical Society of Radiology, the National Consultant for Radiology and Diagnostic Imaging, and the Minister of Health. In addition, the study involved the analysis of information provided on CT referrals by referring clinicians.

Results: Nearly $21 \%$ of CT referrals were found to be unsubstantiated based on the diagnosis made by the referring physician, the body region of interest, and the clinical department. Most referrals identified as non-compliant with the guidelines were related to cancers followed by inflammatory conditions. The lowest number of unjustified exams was reported for endoprostheses and injury-related cases.

Conclusions: The study revealed a significant degree of non-compliance with the diagnostic algorithm as defined in the iRefer guidelines, particularly in cases of cancers and inflammatory conditions. Consequently, the patient's exposure to ionizing radiation is increased. Incorrect decisions regarding the appropriate diagnostic imaging technique are founded on the lack of appropriate cooperation between the clinician and the radiologist, insufficiency or lack of information provided on the referral, as well as the defensive attitude of referring physicians. It is therefore necessary to change appropriate in-hospital management and cooperation models.

Key words: computed tomography, orthopaedics, ionizing radiation, defensive medicine, iRefer.

\section{Introduction}

As the number of diagnostic imaging examinations being performed in patients is increasing, doubts are also increasingly raised regarding their appropriateness and the potential abuse of costly imaging techniques. When involving ionizing radiation, unjustified examinations may also have biological implications. In addition, they have economic consequences and increased waiting times.
All these aspects are particularly relevant in the case of computed tomography (CT).

The aforementioned dynamic development and increased accessibility to individual imaging diagnostic modalities, mainly CT and magnetic resonance imaging (MRI), often leads to patients being referred for diagnostic scans without adequate knowledge of their clinical relevance. Consequently, the diagnostic methods may not be adequate for the clinical problem.

Correspondence address:

Tomasz Latos, Department of Radiology, Medical Centre for Postgraduate Education, Warsaw, Poland, e-mail: tomaszlatos64@gmail.com

Authors' contribution:

A Study design · B Data collection · C Statistical analysis · D Data interpretation · E Manuscript preparation · F Literature search · G Funds collection 
It is well known that medical exposures are subject to certain rules and that the expected health benefits must outweigh any hazard as pursuant to international regulations, in particular to the Council Directive 2013/59/ EURATOM in force within the EU and the revised Nuclear Law Act (Journal of Laws 2019.1792). The emergence of CT at the end of the 1980s brought about a new problem of patients' exposure to ionizing radiation being at times unwarranted. This is against the ALARA principle stating that doses required to achieve the diagnostic benefit should be "as low as reasonably achievable".

Among other factors, the problem of questionable validity of imaging studies is due to non-compliance with clinical indications for diagnostic imaging studies in general, or CT in this instance. Clinical indications for CT and other imaging studies have been published for many years by the Royal College of Radiologists (RCR) and the International Atomic Energy Agency (IAEA) in Europe as well as by the American College of Radiology (ACR) in the US. In Poland "Guidelines for Physicians Issuing Diagnostic Imaging Referrals" were published in 2019 by the National Centre for Radiation Protection in Health Care in cooperation with, e.g., the National Consultant for Radiology and Diagnostic Imaging and the National Consultants for Nuclear Medicine and Radiation Therapy, together with the Polish Medical Radiology Association. This is the eighth edition of the Polish translation of iRefer, i.e. guidelines developed by British experts from various academic centres (RCR) following the analysis of thousands of diagnostic imaging studies. The paper and the electronic version of this document should support clinicians and radiologists when determining the appropriate diagnostic algorithm. The premise of the Guidelines is to facilitate the choice of the most appropriate imaging modality in a variety of clinical situations.

In addition to the hierarchical order of recommended exams, the document provides evaluative recommendation grades of $\mathrm{A}, \mathrm{B}$, and $\mathrm{C}$, depending on the level of experience and the clarity of the recommendations. The guidelines are based on the certainty of imaging evidence as defined according to the level of evidence established for primary studies by the Oxford Centre for Evidence-Based Medicine and the "Journal of Bone and Joint Surgery". Recommendations are graded according to the evidence level. The guidelines make use of recommendation grades as defined by the Oxford Centre for EvidenceBased Medicine. The recommendation grade corresponds to the highest level of evidence relevant to the particular clinical problem. In many cases, grade $\mathrm{B}$ or $\mathrm{C}$ refers to the available evidence base rather that to the relevance of particular recommendations for the clinical problem.

In light of the studies conducted to date, CT scans non-compliant with the Guidelines and scans that could successfully be replaced by other diagnostic modalities (X-ray, ultrasound, MRI) were classified as inappropriate. Scans that did not contribute to the overall diagnostic process, i.e. to correct diagnosis or exclusion of a disease, or had no impact on patient management, were also classified as inappropriate. Scans for which no benefit outweighing the potential negative health effects could be demonstrated were also classified as inappropriate.

It is estimated that the percentage of unwarranted and clinically useless CT scans for all organ groups is usually between 6 and 30\%, depending on factors such as the experience of the study centre, the body region, methodology, lack of appropriate knowledge of the indications to CT, and others. Among the first detailed reports and attempted analyses of the appropriateness of CT scans were those by Oikarinen, Lehnert, Lammers, and Brenner.

According to Brenner, at least $25 \%$ of CT scans could have been replaced by another modality or even abandoned completely [1]. In addition, note should be taken of relevant information not being communicated on the referrals for a CT scan. Referrals are often laconic, lacking substantiation and additional information required for the study to be performed according to an appropriate protocol.

These aspects contribute to the excessive use of costly diagnostic procedures involving the use of ionizing radiation and contrast agents.

The authors have attempted to evaluate the causes of unwarranted orthopaedic CT scans, to analyse the indications for osteoarticular CT scans against selected guidelines as used in other countries (UK, US), and to establish the causes of communication errors occurring between the orthopaedist and the radiologist, potentially resulting in unwarranted and clinically useless examinations being performed.

\section{Material and methods}

The study involved a retrospective review of lower-extremity CT scans acquired in 1410 patients of a renowned orthopaedic hospital in the years 2014-2018. The age of the subjects ranged from 3 to 90 years. The median age was 56 years. The number of women was 612 , and the number of men was 798. Referrals were issued by a total of 11 departments. All scans were acquired using multi-row GE Bright Speed Elite and TOSHIBA Aquiton one TSX-301 (320 row) CT systems.

The analysis made use of computer-based exam and report archiving methods with the help of the following software:

- AMMS for viewing medical histories of individual patients;

- RIS Chazon Ver. 1.9.27 rev. 9724 for viewing examination reports;

- Carestream Vue PACS for viewing individual CT scans.

All studies were evaluated for compliance with the Guidelines (iRefer) providing a validated diagnostic algorithm based on years of experience. The scans and their appropriateness were evaluated by 2 radiologists; in selected questionable cases the diagnostic algorithm was consulted 
with an experienced orthopaedist. After the referral information was assessed for completeness, justification of referrals was compared to that proposed in the Guidelines (iRefer). In addition to the recommendation grade (A to C), as defined in the MS section of the Guidelines and comprising the mainstay recommendation for the assessment of examination validity, the experts based their evaluation on their own experience, possibly as corroborated by the opinion of an orthopaedist. The pooled data were subjected to statistical analysis.

The economic impact of the misdecisions regarding the use of CT diagnostics due to its being inadequate for clinical diagnosis was not taken into account in this study.

Referrals were analysed for information provided, including the following:

- the diagnosis;

- the objective of the exam;

- the rationale;

- and additional information required to carry out the exam.

Included in the analysis were CT studies performed between 14.01.2014 and 31.12.2018. A file listing patients' referrals for CT scans was used in order to determine factors affecting the appropriateness of referrals. The referrals pertained to scans of the lower limbs, hip joints, knee joints, ankle joints, thighs, lower legs, and feet. The referrals were issued by physicians affiliated with 11 departments within the orthopaedic hospital. For the purpose of the analysis, the departments were numbered 1 through 11 (O1 through O11). Patients were referred for CT studies from all these departments, albeit in different proportions depending on the department's clinical profile. The database contained a total of 1410 records corresponding to 1410 exams.

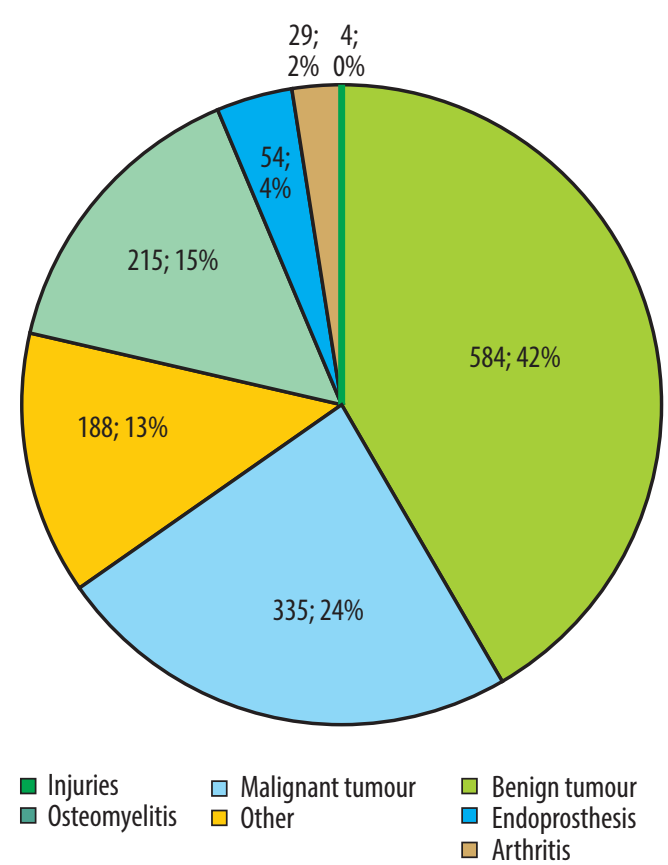

Figure 1. Graphical distribution of initial diagnoses as provided in computed tomography referrals
Statistical analyses were carried out in 2 stages. In the first stage, descriptive statistics of the analysed variables were made and the influence of selected variables on the legitimacy of research conducted using basic analyses was assessed. In the second stage, a holistic model was built, which shows the total impact of the analysed variables on the validity of the CT study (model analysis). STATISTICA 13 (software) was applied for basic analysis, and gretl (software) was used for model analysis. The basic and model analyses were complementary.

\section{Results}

Following examination of $1410 \mathrm{CT}$ exams, 1119 referrals, corresponding to $79.66 \%$ of the total number, were considered justified. A total of 291 referrals, corresponding to $20.64 \%$ of the total number, were considered unjustified (Figures 1-3). 30\% of CT referrals indicated the objective of the examination. $33 \%$ of CT referrals included justification for the referral. $31 \%$ of CT referrals provided information relevant for the CT scan.

The results of multiple comparisons are summarized in Table 1. For the diagnosis of the endoprosthesis for which the percentage of justified CT referrals was the largest (93\%), statistically significant differences were observed as compared to the diagnoses of other, osteomyelitis, benign tumour, and malignant tumour. For the diagnosis of malignant tumour, $0 \%$ of referrals were justified, with statistically significant differences being observed as compared to the diagnoses of endoprosthesis, injuries, and other. However, this outcome might have been affected by a very small number of malignant tumour diagnoses.

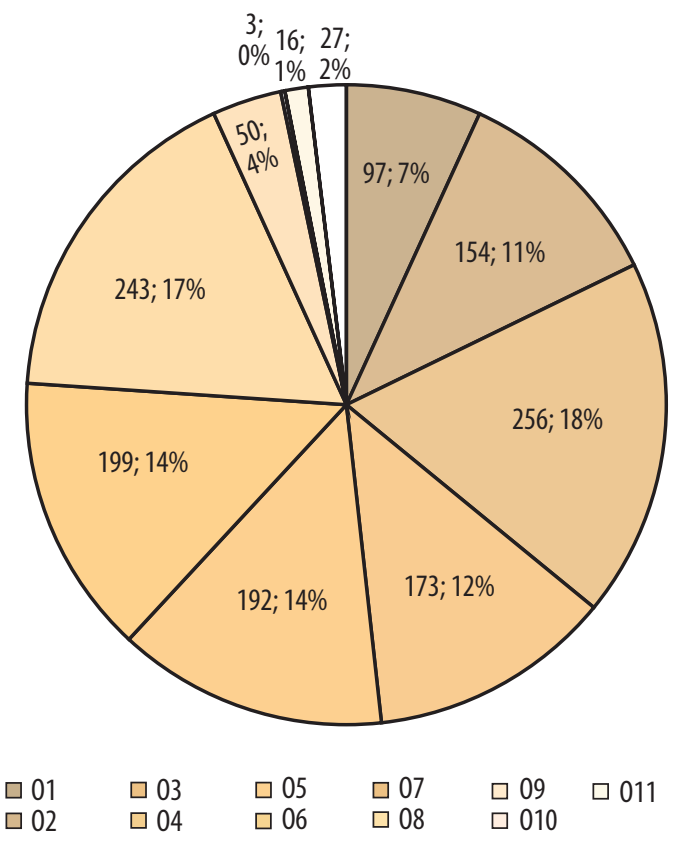

Figure 2. Percentage of computed tomography referrals from individual departments 


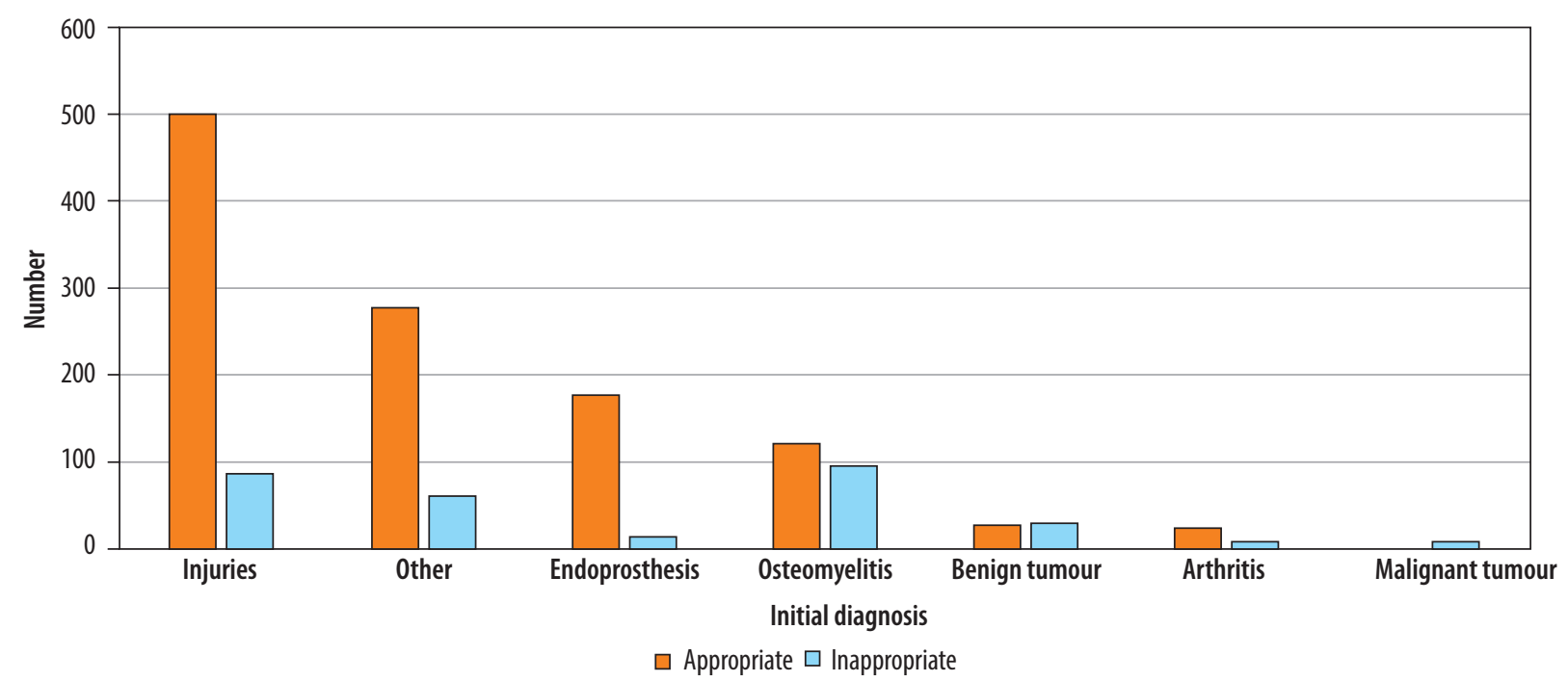

Figure 3. The appropriateness of computed tomography exams by initial diagnosis as provided in the referral. Columns are headed with percentages of appropriate and inappropriate exams being ordered for a particular indication

Table 1. Statistical values for the multiple comparison $\chi^{2}$ test. Statistical values should be compared against the adjusted significance level $\alpha=0.0024$

\begin{tabular}{|c|c|c|c|c|c|c|c|}
\hline & Injuries & Other & Endoprosthesis & Osteomyelitis & Benign tumour & Arthritis & Malignant tumour \\
\hline Injuries & * & 0.227 & 0.006 & $<0.001$ & $<0.001$ & 0.364 & $<0.001$ \\
\hline Other & & * & $<0.001$ & $<0.001$ & $<0.001$ & 0.865 & $<0.001$ \\
\hline Endoprosthesis & & & * & $<0.001$ & $<0.001$ & 0.036 & $<0.001$ \\
\hline Osteomyelitis & & & & * & 0.210 & 0.016 & 0.086 \\
\hline Benign tumour & & & & & * & 0.004 & 0.200 \\
\hline Arthritis & & & & & & * & 0.007 \\
\hline Malignant tumour & & & & & & & * \\
\hline
\end{tabular}

Figure 4 presents the numbers of justified vs. unjustified referrals by referring departments. Columns are headed with percentages of appropriate and inappropriate exams being ordered by a particular department. The referring unit (department) determined the clinical profile, i.e. the type of relevant disorder. The analysis of the material revealed a significant relationship between the percentage of justified exams and the referring unit.

A significant relationship was observed between the referring unit and the appropriateness of the CT referral $\left(\chi^{2}=97.62, p<0.001\right)$. The results of multiple comparisons are summarized in Table 2 . The percentage of justified exams for department O6 ( $94 \%$ of the justified exams) was significantly higher than the percentages of justified exams for departments O1 (74\% of justified exams), O2 (81\% of justified exams), O4 (77\% of justified exams), O7 (60\% of justified exams), O8 (74\% of justified exams), and $\mathrm{O} 10$ ( $56 \%$ of justified exams). On the other hand, a low percentage of justified exams was reported for department $\mathrm{O} 7$ ( $60 \%$ of justified exams), its value being lower than that for $\mathrm{O} 2, \mathrm{O} 3, \mathrm{O} 4, \mathrm{O} 5, \mathrm{O} 6$, and $\mathrm{O} 11$. An even lower percentage of justified exams was reported for department O10, but a statistically significant difference could be observed only as compared to O6 due to the low department size.
The resulting logistic regression model proved to be statistically significant; certain variables were found to impact the validity of the CT scans $\left(\chi^{2}=208.511, p<0.001\right)$. In addition to the generated values of the $\beta$ coefficient ( $\beta$ ), standard error (SE), test statistics $(Z)$, and test probability value $(p)$, an "impact on $\mathrm{H}$ " (factor's influence on the hypothesis) variable was added to indicate how each of the individual variables affected the appropriateness variable $\left(^{*}\right.$ - low impact, ${ }^{* *}$ - moderate impact, ${ }^{* *}$ - significant impact), and the odds ratio (OR, \%) was calculated from the $\beta$ coefficient according to the following formula: odds ratio $=\mathrm{e}^{\beta}$. The odds ratio was used to determine the impact of the individual variables on the appropriateness variable, showing the percentage by which the chance of a justified CT scan increased or decreased for a particular variable.

Impact on $\mathrm{A}=(1-\mathrm{OR}) \times 100 \%$

Positive and negative values corresponded to increased and reduced appropriateness of the exam, respectively.

No difference was observed in the length of the physician's experience between the groups of justified and unjustified exams $(Z=-0.89, p=0.297)$. As seen in Figure 5, the largest percentages of referring physicians issuing referrals for justified as well as unjustified examinations had 1-10 and 11-20 years of experience. 


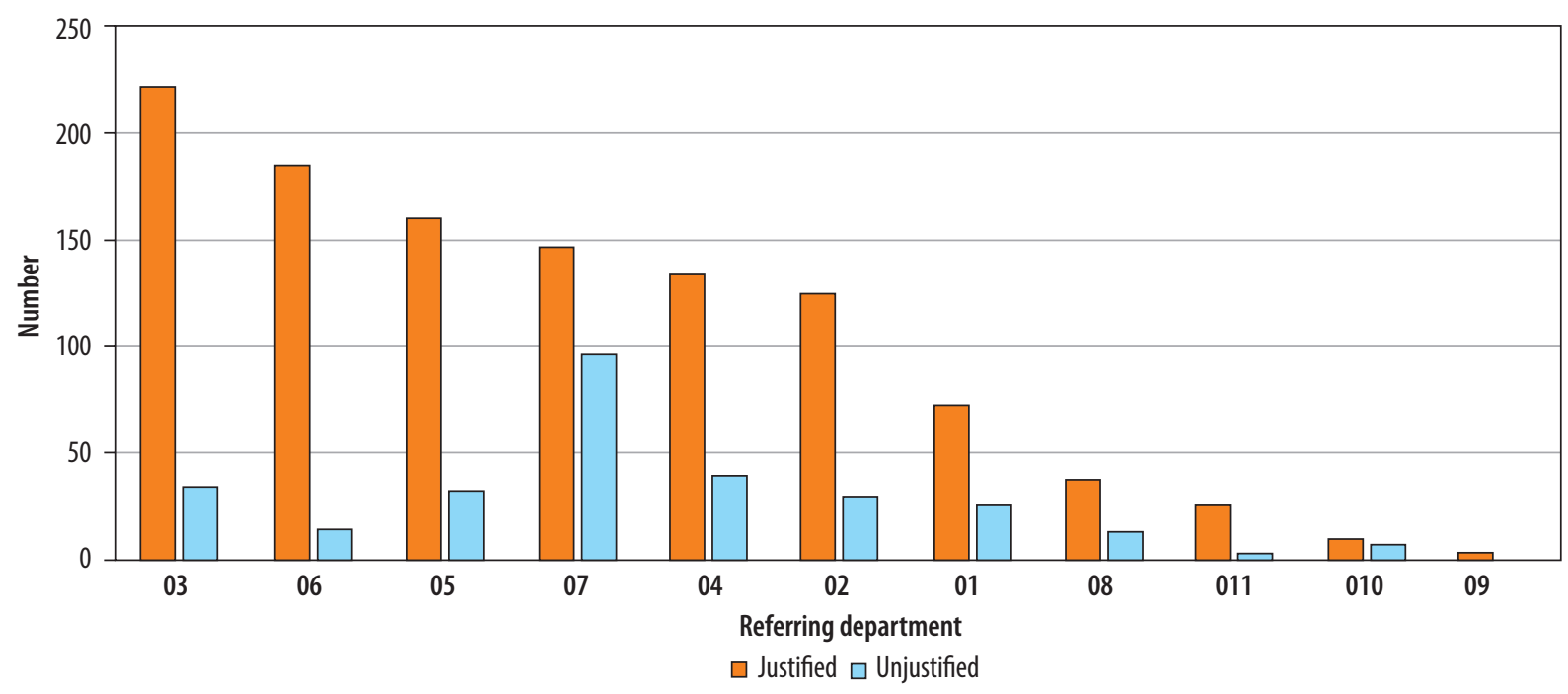

Figure 4. Appropriateness of computed tomography studies by the referring unit. Columns are headed with percentages of appropriate and inappropriate exams being ordered by a particular referring department

Table 2. Statistical values for the multiple comparison $\chi^{2}$ test. Statistical values should be compared against the adjusted significance level $\alpha=0.001$

\begin{tabular}{|c|c|c|c|c|c|c|c|c|c|c|c|}
\hline & 01 & 02 & 03 & 04 & 05 & 06 & 07 & 08 & 09 & 010 & 011 \\
01 \\
02 & $*$ & 0.192 & 0.05 & 0.549 & 0.066 & $<0.001$ & 0.017 & 0.976 & 0.735 & 0.238 & 0.075 \\
03 & & $*$ & 0.131 & 0.409 & 0.600 & $<0.001$ & $<0.001$ & 0.276 & 0.935 & 0.045 & 0.240 \\
04 & & & $*$ & 0.012 & 0.317 & 0.031 & $<0.001$ & 0.022 & 0.855 & 0.003 & 0.570 \\
05 & & & & & $*$ & 0.003 & $<0.001$ & 0.131 & 0.990 & 0.020 & 0.337 \\
06 & & & & & & $*$ & $<0.001$ & $<0.001$ & 0.503 & $<0.001$ & 0.742 \\
07 & & & & & & & $*$ & 0.072 & 0.424 & 0.737 & $<0.001$ \\
08 & & & & & & & & $*$ & 0.744 & 0.301 & 0.049 \\
09 & & & & & & & & & $*$ & 0.430 & 0.464 \\
010 & & & & & & & & & & $*$ & 015 \\
\hline 011 & & & & & & & & & & & $*$ \\
\hline
\end{tabular}

01-011-department no. 1-11

Positive and negative values corresponded to increased and reduced appropriateness of the exam, respectively (Table 3).

Following the analysis of data in the "impact on A" and "OR" columns, the following factors were observed to increase the appropriateness of CT exam referrals:

- physician issuing the referral (linked to the clinical profile of the department);

- CT exam to be performed in a patient with endoprosthesis (referrals nearly 3 times more likely to be justified);

- patients with known injuries (the probability of a justified exam increased by $55.3 \%$ ).

In conclusion, following the overall assessment of the appropriateness or inappropriateness of CT scans performed, it is concluded that $79.36 \%$ of all analysed CT exams (regardless of the department or clinical diagnosis) were considered justified, as opposed to $20.64 \%$ of exams that were deemed unjustified.

\section{Discussion}

Following the analysis of 1410 CT examinations, a total of 291 exams corresponding to $20.64 \%$ of the total number were found to be unjustified. This result is similar to the one presented in recent publications from different medical centres worldwide. The problem of non-compliance with the Guidelines (iRefer) is probably due to various reasons, as set out in several publications. In our study material, clear correlation could be observed with clinical diagnosis. For endoprostheses and injuries, the percentage of unjustified exams was small and amounted to 6.91\% and $14.55 \%$, respectively. Conversely, initial diagnoses of malignant tumours, benign tumours, or inflammatory conditions were associated with the highest percentages of unjustified exams, which amounted to $56.90 \%$ for tumours (including $53.70 \%$ for benign tumours) and $44.19 \%$ for osteomyelitis. Data for malignant tumours may be 


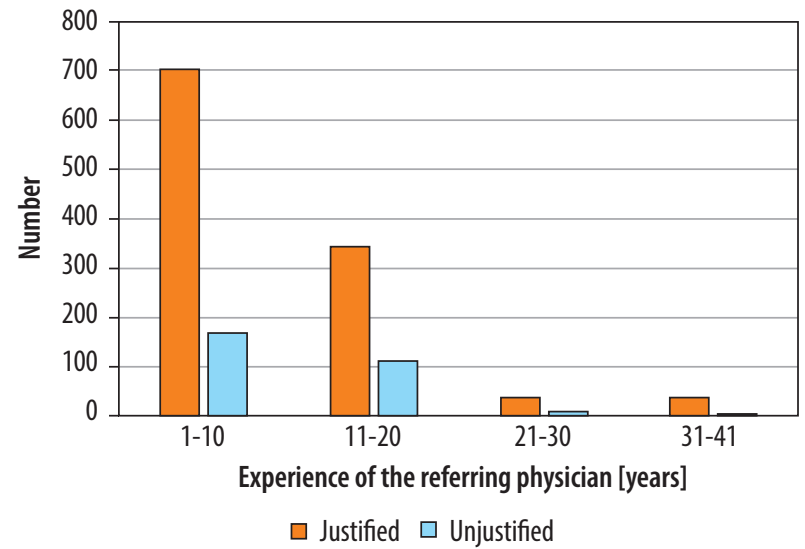

Figure 5. The appropriateness of computed tomography exams by the length of experience of the referring physician. Columns are headed with percentages of appropriate and inappropriate exams being ordered by physicians with particular length of experience

misleading due to a low number of records. For arthritis, the percentage of unjustified referrals was close to that in the entire study sample and amounted to $20.69 \%$. Closer collaboration between the clinician and the radiologist could contribute to appropriate adjustments to the diagnostic algorithm. An interesting aspect of the study consisted of the analysis of referral completion. No impact on increased percentage of justified referrals was observed for the lack of rationale or the lack of examination procedurerelevant information. Paradoxically, failure to provide an examination objective had a positive effect on the number of justified studies. This indicates that compliance with the proper diagnostic algorithm as set out in the Guidelines and precise reasons for the referral being provided to the radiologist are 2 altogether different issues. However, the failure to provide accurate referral information undoubtedly can affect the quality of the radiological exam summary because less information is available to the examining physicians. Of course, physicians make use of the hospital's IT systems and may analyse the patient's medical history, but it is nonetheless very important to ensure better cooperation between the clinician and the radiologist, as mentioned before. No effect of the length of the referring physician's experience on the appropriate-

Table 3. Results generated by the program with limit value analysis

\begin{tabular}{|c|c|c|c|c|c|c|c|}
\hline Name & $\beta$ & SE & $Z$ & $p$ & Impact on $\mathrm{H}$ & $\mathrm{OR}$ & Impact on A \\
\hline const & 2.36 & 0.30 & 7.82 & $<0.001$ & $* * *$ & 10.59 & \\
\hline 01 & -0.80 & 0.38 & -2.11 & 0.035 & $* *$ & 0.45 & $-55.0 \%$ \\
\hline 02 & -0.66 & 0.35 & -1.86 & 0.063 & * & 0.52 & $-48.3 \%$ \\
\hline 03 & -0.88 & 0.34 & -2.62 & 0.009 & $* * *$ & 0.42 & $-58.5 \%$ \\
\hline 04 & -1.42 & 0.32 & -4.42 & $<0.001$ & $* * *$ & 0.24 & $-75.7 \%$ \\
\hline 05 & -1.13 & 0.31 & -3.58 & $<0.001$ & $* * *$ & 0.32 & $-67.6 \%$ \\
\hline 07 & -0.81 & 0.48 & -1.70 & 0.090 & * & 0.44 & $-55.7 \%$ \\
\hline 010 & -3.05 & 0.76 & -4.00 & $<0.001$ & $* * *$ & 0.05 & $-95.2 \%$ \\
\hline L14 & -1.09 & 0.38 & -2.85 & 0.004 & $* * *$ & 0.33 & $-66.5 \%$ \\
\hline L16 & -2.32 & 0.71 & -3.29 & 0.001 & $* * *$ & 0.10 & $-90.2 \%$ \\
\hline L17 & -0.73 & 0.37 & -1.96 & 0.050 & $* *$ & 0.48 & $-52.0 \%$ \\
\hline L26 & -1.23 & 0.50 & -2.46 & 0.014 & ** & 0.29 & $-70.8 \%$ \\
\hline$\angle 43$ & 2.91 & 1.34 & 2.17 & 0.030 & $* *$ & 18.34 & $1734.4 \%$ \\
\hline L51 & -1.14 & 0.68 & -1.68 & 0.093 & * & 0.32 & $-68.1 \%$ \\
\hline L54 & -2.33 & 1.30 & -1.79 & 0.074 & * & 0.10 & $-90.2 \%$ \\
\hline L61 & -1.50 & 0.62 & -2.41 & 0.016 & $* *$ & 0.22 & $-77.6 \%$ \\
\hline L77 & -1.64 & 0.86 & -1.91 & 0.056 & * & 0.19 & $-80.6 \%$ \\
\hline L78 & -0.54 & 0.29 & -1.82 & 0.069 & * & 0.59 & $-41.5 \%$ \\
\hline L90 & -1.05 & 0.49 & -2.17 & 0.030 & $* *$ & 0.35 & $-65.2 \%$ \\
\hline L101 & -3.44 & 1.30 & -2.65 & 0.008 & $* * *$ & 0.03 & $-96.8 \%$ \\
\hline$E$ & 1.04 & 0.32 & 3.27 & 0.001 & $* * *$ & 2.83 & $183.4 \%$ \\
\hline NN & -1.87 & 0.33 & -5.68 & $<0.001$ & $* * *$ & 0.15 & $-84.6 \%$ \\
\hline U & 0.44 & 0.23 & 1.90 & 0.057 & * & 1.55 & $55.3 \%$ \\
\hline Omye & -1.07 & 0.40 & -2.66 & 0.008 & $* * *$ & 0.34 & $-65.9 \%$ \\
\hline
\end{tabular}

$\beta$ - the strength of the "Impact on A" coefficient

0 - Department, L - Physician, E - Endoprosthesis, NN - Benign tumour, U - Injuries, Omye - 0steomyelitis 
ness of referrals was observed. On the other hand, statistically significant differences were observed for individual department units.

According to the author's observations, this was primarily related to the unit's profile, which translated into the number of hospitalized patients with specific initial diagnoses. This in turn determined the number of unjustified referrals being issued without compliance to the algorithm set out in the Guidelines; this pattern could be observed for physicians with both short and long professional experience. Obviously, differences in this regard could be tracked for individual physicians; for example, the chance of a justified referral being issued by physician no. 43 was 18 times higher than the reference level, whereas nearly all referrals issued by physician no. 101 were considered unjustified. A statistically significant difference was also observed between the groups of justified and unjustified exams in relation to patients' age. The median age was higher in the case of justified exams. Because patients with endoprostheses were almost three times more likely to present with justified referrals, the correlation with age appears obvious.

In recent years, similar problems were investigated by research groups from different countries, e.g. Finland and the US. This led to similar conclusions suggesting that imaging studies, especially those involving ionizing radiation, should be subject to precise algorithms [1-7]. Because these examinations play an increasingly important role in the diagnostic process, they tend to be abused and used without justification or according to improper algorithms. Frequently, awareness of the consequences of exposures to ionizing radiation is lacking [1-4,8-10]. Avoiding such exposures would not only lead to savings due to the abandonment of unnecessary tests, but above all would be in line with the principles of radiological protection against ionizing radiation. Also, the number of referrals for diagnostic imaging studies is too high due to the overcautious attitude of some physicians concerned about potential claims from their patients. This problem is increasingly common in Poland, as observed by the author in his analysis of individual CT referrals.

A similar phenomenon was previously recognized and nicknamed "defensive medicine" by some American physicians [11-13]. Hendee et al. [14] estimated that the costs of "defensive medicine" can be as high as $\$ 1.4$ billion per annum in one state alone. This problem is probably greater in the US as compared to other countries due to a more "predatory" legal climate. Many observers assume that the expenditures on defensive medicine fall between $5 \%$ and $25 \%$ of the total costs of diagnostic imaging studies performed in the United States.

In order to avoid the aforementioned problems, the Ministry of Health published the "Guidelines for Physicians Issuing Diagnostic Imaging Referrals", the $8^{\text {th }}$ edition of which was issued in Poland in 2019. The document is a Polish version of the Royal College of Radiologists'
"RCR iRefer Guidelines: Making the Best Use of Clinical Radiology, $8^{\text {th }}$ Edition". The guidelines are recommended by the Ministry of Health; a free copy of the guidelines should be used by physicians issuing diagnostic imaging referrals on a day-to-day basis.

One of the most spectacular examples of the inappropriateness of CT scans was published by Oikarinen et al. [2]. The analysis pertained to studies performed over a period of several years at the University Hospital in Oulu, Finland. A study carried out in a group of patients below the age of 35 years revealed that as many as $77 \%$ of all CT scans of the lumbar spine region were unjustified. According to the authors who analysed all outcomes on a caseby-case basis, all these unjustified exams should have been replaced by MRI scans. Equally spectacular results applied to CT scans of the head as well as of the abdomen and pelvis minor, with $36 \%$ and $37 \%$ of the exams being found to be unjustified, respectively. These should have been replaced by MRI or US scans. Only 3\% of unjustified studies were identified for the cervical spine segment. The authors stressed that the exposure to ionizing radiation, particularly in the case of the lumbar spine and the abdomen, was very high and amounted to the equivalent of 170 and 500 chest X-rays, respectively. Notably, the CT exams included in the study had been acquired in patients below the age of 35 years, i.e. in patients of reproductive age. The authors of the publication also reported on the increase in the number of exams performed in the following years and assumed that the growing trend would continue. Therefore, priority should be given to reducing the number of unjustified referrals for CT exams. The appropriateness of referrals was found to be higher for emergency as compared to elective procedures.

This paper analysed the referrals issued within a large orthopaedic hospital; however, non-compliance with appropriate algorithms can be observed in literature reports pertaining to the outpatient setting and primary health care as well. Lehnert et al. [3] analysed the appropriateness of CT and MRI examinations performed pursuant to referrals issued by primary care physicians. On average, $26 \%$ of studies were considered to be unjustified, with the highest percentage of questionable CT referrals being related to the spine (53\%) as well as the head in cases of poorly justified chronic pain conditions (62\%). The authors highlight that the established examination referral guidelines and correct diagnostic algorithms are too rarely used by primary care physicians, who often have little time to examine their patients. An important element underlined in the work and referring to the earlier publications by Brenner and Hall [7] is the risk of cancer induction, which should always be borne in mind by physicians referring their patients to imaging studies involving high doses of ionizing radiation. These stochastic effects, frequently overlooked in day-to-day medical practice, are the main reason behind the requirement to follow the Guidelines, and they provided the most important inspiration 
for this study. In light of the above, the high percentage of unjustified referrals of patients initially diagnosed with cancer is all the more worrying.

In another of their publications, Hall and Brenner [15] note that the use of CT has increased 12 fold in the UK and 20 fold in the US since the early 1980s. In the US, the average effective dose from all studies involving $\mathrm{X}$-rays increased 7 fold during this period. Thus, exposure to ionizing radiation for medical purposes constitutes the majority of the effective radiation dose received by individuals in the United States. Among other factors, this is because CT is a fast, simple, and accurate diagnostic tool. Once again, the authors recall the need to apply the ALARA principle.

According to Hricak [16], ionizing radiation for medical purposes accounts for $95 \%$ of total exposure from human-made sources and approximately half of the total radiation exposure in the US. Between 1995 and 2010, the number of CT studies grew by $10 \%$ per year, with the US population growing at a rate of less than $1 \%$ per year.

Gransjøen et al. [11] analysed the reasons behind misreferrals for imaging studies by conducting interviews with 8 general practitioners and 10 radiologists from 2 administrative regions in Norway. Several reasons leading to non-compliance with the proper diagnostic algorithm were identified. These included, in particular, limited examination time, pressure from patients, and guidelines being too long or unclear for the referring physician. Factors contributing to the increased ease of use of the guidelines included their ready availability and adaptation to the target audience. Other factors influencing the decisions to refer patients to diagnostic imaging studies consisted of the availability of these studies as well as symptoms and the number of visits the patient had with the family physician.

To quote one of the opinions, "[...] instead of spending an hour arguing with the patient whether a particular examination is indeed necessary, you just write a referral; it takes two minutes and you're done [...]" [11]. This shows how important it is to inform patients about the potential risks arising from the use of diagnostic methods involving ionizing radiation $[17,18]$. According to the responders, outsourcing of unjustified examinations was also due to the need for profit in privately-owned institutions. The interviewed physicians claimed that there were too many guidelines and not enough time to keep up with all of them. Another factor was the fact that some guidelines did not have a high priority, which also influenced the decisions being made [11].

In this study, CT exams were ordered despite the recommendation for CT being low while simultaneously being high for magnetic resonance imaging. In 2001, Brenner et al. $[19,20]$ estimated the risk of cancer caused by CT examinations in children to recognize that CTinduced cancer may be the cause of death of up to 500 people. Of course, these estimates are the result of a statistical analysis comparing the incidence of cancer depend- ing on the exposure or non-exposure to ionizing radiation as used in CT examinations.

According to Pearce et al. [21], a greater incidence of brain cancer and leukaemia can be demonstrated in children and adolescents who have undergone a CT exam. The authors analysed CT examinations performed in the years 1985-2002 in various hospitals in the UK. The average duration of follow-up was slightly below 10 years. According to the authors' estimates, 10,000 head CT scans could be responsible for one excess case of brain cancer in relation to the populational probability. It is important to remember that there is no threshold dose for the stochastic effects of radiation. These data demonstrate the need to minimize CT exams of questionable validity. The increased risk of cancer caused by ionizing radiation was also addressed in other publications [20,22,23]. Therefore, the need for follow-up CT scans in oncological diagnostics should be carefully considered each time [24].

In the material collected for the purposes of this study, exams deemed unjustified were mainly due to the use of an incorrect diagnostic algorithm following a particular clinical diagnosis or suspicion of a disease. No such correlation was found for referrals lacking the rationale or examination procedure-relevant information. Paradoxically, no increase in the percentage of unjustified exams was observed when no study objective was provided in the referral, although interpretation of study results was more difficult in these cases. However, incomplete referrals that contain only the diagnosis and no rationale or other details contribute to the increased use of incorrect examination protocols, or they make it more difficult to correct the algorithm proposed by the clinician. An interesting study was published by Brenner [1]. The total number of CT studies performed in the year 2012 was 85 million in the US as compared to 3 million in the UK. The annual rate of growth in the use of CT was $6.5 \%$ in the US and $9.4 \%$ in the UK. Paediatric CT studies account for between 5 and $10 \%$ of the total number of CT studies performed in the US. In the year 2000, the International Commission on Radiological Protection (ICRP) concluded that the dose absorbed by tissue from a CT scan may often approach or exceed levels known to increase the likelihood of cancer. We should remember that the concerns regarding the use of CT relate mainly to population and public health risks (low risk multiplied by a large population at risk), and not to individual risks.

Different methods are also available for reducing or at least inhibiting the increase in radiation dose resulting from CT use. The first is to reduce the used dose by using stateof-the-art CT scanners. Another is to replace CT with other imaging modalities according to the CT algorithm and to minimize the number of examinations performed when possible.

Reduction of the dose can be achieved, e.g., by manual adjustment of mAs settings for people of different body sizes or by automatic modulation of currents [25]. Preferably, however, other imaging methods, particularly MRI and 
US, should be used in situations provided for in the Guidance. Brenner [1] estimates that it is possible to reduce the number of CT studies by at least $25 \%$ by using appropriate procedures. The possibility of CT scans being abandoned is reduced by the availability of MRI scans, the time required to take the scan, as well as, quite frequently, pressure from patients or patients' parents. These issues were addressed in the articles by Hendee et al. [14] and Hricak et al. [16]. Brenner draws attention to the assessment of compliance with the ACR guidelines, as performed by Hadley et al. [26] in trauma patients. As shown in the analysis, as many as $44 \%$ of CT studies would not have been performed had the ACR adequacy criteria been followed. This shows that proper education in this field is required already at the beginning of the young physician's training [27].

When conducting such analyses, consideration should be given to the level of awareness of both the referring physicians and those performing the exam. In fact, the existing established Guidelines are intended for use not only by the referring physicians; the requirement of adhering to recommendations pertains to those performing the exams worldwide. In Poland, pursuant to nuclear legislation in force, an exam must be passed every 5 years to document appropriate knowledge of the exposures to ionizing radiation, dose levels, and radiation levels appropriate for various X-ray studies. Not all countries have mandated such requirements, and the failure to systematically renew and expand one's knowledge in the field may lead to a much more liberal approach to ionizing radiation exposures. This phenomenon was analysed by Subramniyan Ramanathan and John Ryan [8] from the University Hospital in Ottawa (Ontario, Canada). The researchers carried out a survey among the staff of the hospital's radiology department. The study revealed insufficient knowledge, with an average score of 8.5 out of 17 points. In this context, it is worth recalling a paper by Tahvonen, Oikarinen et al., who re-examined the appropriateness of CT referrals in young adults and children. According to the authors, it appears that the number of justified studies has increased by $16 \%$ as compared to the previous study, as a result of educational measures, including several 3-hour training courses, being undertaken, with the most radical increase being observed for spine CT scans. This demonstrates the need for the proposed wider education regarding the need to comply with the guidelines.

From the point of view of healthcare managers, the economic aspects of the abuse of imaging studies, particularly CT scans, are also worth mentioning. Although not presented in this work, such an analysis is recommended for future studies. The ACR adequacy criteria may have a significant impact on the imaging costs as well as on the dose of the ionizing radiation delivered. This was addressed in an article by Hadley et al. [26], who estimated that the total treatment cost in a group of patients could have been reduced by $39 \%$ by abandoning 257 CT examinations, with a total saving of $\$ 325,377$. According to the authors' estimates, compliance with ACR criteria when performing imaging studies in trauma patients in the US has the potential to save billions of dollars. In addition, as mentioned by other, previously cited authors, Hadley et al. believe that eliminating unjustified CT studies would reduce cancer morbidity by several thousand cases per year.

The costs associated with the use of modern imaging techniques are highlighted by Dick et al. [28]. The authors provide data and an assessment of the consequences of excessive and exaggerated diagnostic processes that increase the duration of ED stays and indirectly increase the risk of medical errors due to overfilling. An efficacy and cost analysis was also carried out by Renton et al. [29]. This is the main reason for complaints filed by patients attending emergency departments at Polish hospitals.

Tung et al. [13], having examined the reasons for unjustified CT referrals, state that besides non-compliance with the guidelines, these reasons include fear of an alternative, rare disease not being taken into account and the consequences of potential misdiagnosis. Other arguments included pressure from patients or their families, standard practices within a particular medical environment not being in line with the established guidelines, the emergency medicine operation model, and administrative pressure related to the financing of medical examinations or personal benefits from such financing.

In Poland, the problem of unjustified referrals for CT studies was addressed by Sobiecka et al. [9] as well as by Wierzchołowski et al. [10] and the author of this study. The problems highlighted by the authors of papers mentioned above from various countries have been confirmed in the material analysed in Poland. Failure to follow the "Guidelines" is the fundamental reason behind unjustified referrals, and the previously mentioned lack of cooperation between the clinician and the radiologist makes the application of correct diagnostic algorithms more difficult. Accurate information provided in the referral would not only help the radiologist in summarizing the study results but also facilitate a different diagnostic algorithm being proposed. For this reason, such cooperation is very important. Changing this situation to reduce the patients' exposure to ionizing radiation as well as the unnecessary costs of examinations is a challenge both for health care professionals and health care managers.

\section{Conclusions}

The number of unjustified CT studies is similar to those observed in studies performed at different sites worldwide.

The type of pathology identified in the referral affected the frequency of unjustified exams, with the highest percentage of unjustified referrals being observed for osteomyelitis and tumours.

The initial diagnoses of endoprosthesis and trauma were associated with the highest percentage of justified studies. 
Statistically significant differences in the accuracy of the diagnostic algorithms were observed between different departments, mainly due to differences in the incidence of particular pathologies, which were in turn linked to the specialization of different departments.

No correlation was observed between the rationale, objective, or examination-relevant information being provided in the referral and the appropriateness of the exam.

The length of the clinician's experience did not correlate with the appropriateness of the referral.
At the same time, significant differences were observed between the numbers of justified referrals being issued by individual physicians from different departments. Wider promotion of knowledge on the Guidelines (iRefer) and the necessity of compliance therewith is required.

\section{Conflict of interest}

The authors report no conflict of interest.

\section{References}

1. Brenner DJ. Minimising medically unwarranted computed tomography scans. Ann ICRP 2012; 41: 161-169.

2. Oikarinen H, Meriläinen S, Pääkkö E, et al. Unjustified CT examinations in young patients. Eur Radiol 2009; 19: 1161-1165.

3. Lehnert BE, Bree RL. Analysis of appropriateness of outpatient CT and MRI referred from primary care clinics at an academic medical center: how critical is the need for improved decision support? J Am Coll Radiol 2010; 7: 192-197.

4. Lammers EJ, Adler-Milstein J, Kocher KE. Does health information exchange reduce redundant imaging? Evidence from emergency departments. Med Care 2014; 52: 227-234.

5. Tahvonen P, Oikarinen H, Pääkkö E, et al. Justification of CT examinations in young adults and children can be improved by education, guideline implementation and increased MRI capacity. Br J Radiol 2013; 86: 20130337.

6. Brenner DJ, Hricak H. Radiation exposure from medical imaging: time to regulate? JAMA 2010; 304: 208-209.

7. Brenner DJ, Hall EJ, Phil D. Computed tomography - an increasing source of radiation exposure. N Engl J Med 2007; 357: 2277-2284.

8. Ramanathan $\mathrm{S}$, Ryan J. Radiation awareness among radiology residents, technologists, fellows and staff: where do we stand? Insights Imaging 2015; 6: 133139.

9. Sobiecka A, Bekiesińska-Figatowska M, Rutkowska M, et al. Clinically unjustified diagnostic imaging - a worrisome tendency in today's medical practice. Pol J Radiol 2016; 81: 325-330.

10. Wierzchołowski W, Walecki JM, Latos T. Rationality of using wholebody computed tomography in trauma patients. Pol J Radiol 2020; 85: e132-e136.

11. Gransjøen AM, Wiig S, Lysdahl KB, Hofmann BM. Barriers and facilitators for guideline adherence in diagnostic imaging: an explorative study of GPs' and radiologists' perspectives. BMC Health Serv Res 2018; 18: 556.

12. Rawle M, Pighills A. Prevalence of unjustified emergency department $\mathrm{x}$-ray examination referrals performed in a regional Queensland hospital: a pilot study. J Med Radiat Sci 2018; 65: 184-191.

13. Tung M, Sharma R, Hinson JS, et al. Factors associated with imaging overuse in the emergency department: A systematic review. Am J Emerg Med 2018; 36: 301-309.

14. Hendee WR, Becker GJ, Borgstede JP, et al. Addressing overutilization in medical imaging. Radiology 2010; 257: 240-245.

15. Hall EJ, Brenner DJ. Cancer risks from diagnostic radiology. Br J Radiol 2008; 81: 362-378.

16. Hricak H, Brenner DJ, Adelstein SJ, el al. Managing radiation use in medical imaging: a multifaceted challenge. Radiology 2011; 258: 889-905.
17. Lee CI, Flaster HV, Haims AH, et al. Diagnostic CT scans: institutional informed consent guidelines and practices at academic medical centers. Am J Roentgenol 2006; 187: 282-287.

18. Lee CI, Haims AH, Monico EP, et al. Diagnostic CT scans: assessment of patient, physician, and radiologist awareness of radiation dose and possible risks. Radiology 2004; 231: 393-398.

19. Brenner DJ, Elliston CD, Hall EJ, Berdon WE. Estimated risks of radiation-induced fatal cancer from pediatric CT. AJR Am J Roentgenol 2001; 176: 289-296.

20. Brenner DJ, Elliston CD, Hall EJ, Berdon WE. Estimates of the cancer risks from pediatric CT radiation are not merely theoretical: comment on "Point/counterpoint: in X-ray computed tomography, technique factors should be selected appropriate to patient size against the proposition". Med Phys 2001; 28: 2387-2388.

21. Pearce MS, Salotti JA, Little MP, et al. Radiation exposure from CT scans in childhood and subsequent risk of leukaemia and brain tumours: a retrospective cohort study. Lancet 2012; 380: 499-505.

22. Brenner DJ, Shuryak I, Einstein AJ. Impact of reduced patient life expectancy on potential cancer risks from radiologic imaging. Radiology 2011; 261: 193-198.

23. Brenner DJ, Doll R, Goodhead DT, et al. Cancer risks attributable to low doses of ionizing radiation: Assessing what we really know. Proc Natl Acad Sci U S A 2003; 100: 13761-13766.

24. Lupichuk S, Tilley D, Surgeoner B, et al. Unwarranted imaging for distant metastases in patients with newly diagnosed ductal carcinoma in situ and stage I and II breast cancer. Can J Surg 2020; 63: E100-E109.

25. McCollough CH, Bruesewitz MR, Kofler Jr JM. CT dose reduction and dose management tools: overview of available options. Radiographics 2006; 26: 503-512.

26. Hadley JL, Agola J, Wong P. Potential impact of the American College of Radiology appropriateness criteria on CT for trauma. AJR Am J Roentgenol 2006; 186: 937942.

27. Amis Jr ES. Noninterpretive skills for radiology residents American College of Radiology Standards, Accreditation programs, and appropriateness criteria. Am J Roentgenol 2000; 174: 307-310.

28. Dick EA, Varma D, Kashef E, Curtis J. Use of advanced imaging techniques during visits to emergency departments - implications, costs, patient benefits/risks. Br J Radiol 2016; 89: 20150819.

29. Renton J, Kincaid S, Ehrlich PF. Should helical CT scanning of the thoracic cavity replace the conventional chest X-ray as a primary assessment tool in pediatric trauma? An efficacy and cost analysis. J Pediatr Surg 2003; 38: 793-797. 